Скопје, Македонија

\title{
ONLINE HOMEWORK SYSTEMS IN CALCULUS
}

\author{
RISTO ATANASOV
}

\begin{abstract}
We will discuss the effectiveness of online homework in $\mathrm{Cal}$ culus courses. Specifically, we will discuss the use of WeBWorK - an open-source online homework system supported by the Mathematical Association of America and the National Science Foundation. We will confer practices for implementing WeBWorK homework for Calculus and discuss student learning using this online homework system.
\end{abstract}

\section{INTRODUCTION}

In the last 10 years, many colleges and universities have opted to use online homework systems for undergraduate students to complete homework assignments. As a form of assessment, online homework systems can certainly save time to the faculty by not grading written homework assignments. However, one can always ask the question do these online systems improve students' learning of mathematics or they harmed the students?

How may the students benefit from using online systems to complete their homework assignments? Here are few arguments that support the use of online homework systems [1].

(1) They provide an instantaneous feedback to the students; the students know if their answer is correct or not and they do not need to wait until they meet their instructor to get a feedback.

(2) The online homework systems usually allow multiple attempts to solve each of the problems. This certainly helps the students learn - if they receive an incorrect answer, then they will either go back to their solution and try to find a mistake or they will use another method to solve the problem.

(3) Students gain confidence in the subject matter and may target the learning more effectively by knowing immediately which problems they are getting marked incorrect.

(4) Most online homework systems use randomized constants in the problems, which means that each student will get a problem with

2010 Mathematics Subject Classification. 97U70.

Key words and phrases. online homework system, WeBWorK, Calculus. 
different constants. This would eliminate copying the answers between the students.

(5) Students may access their assignment using any computer, tablet, or smart phone with an internet connection.

There are also few concerns that might be raised about the online homework systems.

(1) Students may get a correct answer using an incorrect method to solve the problem, or, in some instances, guess the answer. Then they receive a credit for completing the problem without knowing how to solve the problem. This certainly does not help them learn.

(2) Students might make mistakes when typing their solutions; for example, missing parentheses may result in an incorrect answer. In this scenario, the students will spend time looking for a mistake even though they know how to solve the problems.

(3) Ignore the importance of mathematical writing; students are focused on getting the correct answer without paying attention on writing a mathematically correct and precise solution.

(4) Multiple submission may lead to use a trial-and-error strategy.

(5) Students may ask some of their peers to solve the problem for them and they just enter the answer.

The standard written homework assignments (paper and pencil homework) also come with pros and cons. And I am fairly certain, any method we use to assess homework assignments will come with few cons. However, just because a method has cons does not mean that we should give up on it. With few adjustments, it may actually provide the results we are striving for.

There are numerous online homework systems available for the instructors and professors to use in the classroom. WeBWorK, WebAssign, ALEKS, MyMathLab are just few to choose from. However, some of these online homework systems come with the choice of the textbook or they are not free. Western Carolina University, the university where I work, is on a bookrental system - students rent the textbooks at the beginning of the semester and return them when the semester is over; the books are also re-used in the following semesters. Hence, choosing an online homework system that comes with a textbook is not an option for us. We were looking for an online homework system that will help our students learn mathematics, and, at the same time, it will be affordable for the students. The decision was easy - we picked WeBWorK. This online homework system is free and it might be used effectively in the learning process. 


\section{WeBWorK}

References for this section are [2, 3]. WeBWorK is an open-source online homework system for mathematics and science courses. It is supported by the Mathematical Association of America (MAA) and the National Science Foundation (NSF). It comes with a National Problem Library (NPL) of over 20,000 problems that target most lower division mathematics courses (up to multivariable Calculus) and some upper-division courses (Linear Algebra, Probability and Statistics, Differential Equations, etc.).

WeBWorK is used at over 700 universities and colleges ranging from small teaching colleges to large research universities. This online homework system has been developed and maintained since 1994. It provides the mathematical community with the most robust, flexible, and mathematically capable online homework system possible. WeBWorK is freely available. The institutions may download, install, and menage their installation on their own server, or sign up for MAA hosting for a modest fee. The WeBWorK community provides pedagogical and technical help through forums, workshops, minicourses, etc.

Here are some futures of WeBWorK.

(1) Each WeBWorK problem is individualized so that each student has a different version of the problem. For example, one student might be asked to solve the integral $\int e^{2} x \sin (3 x) d x$ and another to solve $\int e^{3} x \sin (4 x) d x$. This future increases the learning process of WeBWorK; a student cannot just copy the answer from another student.

(2) Students may make multiple attempts before answering correctly. The system records the correctness of each attempted answer and the instructors may easily monitor their students progress.

(3) The system is very flexible when entering the answers. The answers do not need to be fully simplified or written in a specific form; the system recognizes many equivalent mathematical expressions. WeBWorK can also handle multiple answering styles, including multiple choice, numerical, graphical, and algebraic input.

(4) Every WeBWork problem has its own feedback button that directly sends an email to the instructor. The instructor can easily check the students' answers and respond to their inquiry.

(5) Students and instructors can easily access WeBWorK interface from any computer connected to the internet and using any browser.

\section{Using WeBWorK in Calculus}

I have been using WeBWorK in my Calculus courses for the past four years. Before this online homework system became a part of my students' 
learning experience and a form of assessment in my courses, I regularly assigned, collected, and graded standard homework assignments. After each class, I assigned 10-20 problems from the textbook, and, by a given due date, the students were asked to submit their solutions. Then I graded a subset of the assignment and provided a written feedback to each student. I usually returned the graded assignments by the next class period bearing in mind that is very important that the students receive a timely feedback on their work. Even with my fast grading pace, it still took at least two days until the students received their feedback. At the same time, the grading and providing feedback was time consuming process for me. However, by grading these standard homework assignments, I focused more one checking and critiquing the whole solution, noticing how well the students understood the concepts discussed in class, and providing suggestions how to improve their mathematical writing.

While re-evaluating my practice of assigning and grading standard homeworks, some of my colleagues started using WeBWorK in their classes. I must admit, I was reluctant of this idea at first. I was aware of all the futures that WeBWorK provides, and I liked many of them. I particularly liked that students receive an immediate feedback when working on WeBWorK problems; if their answer is not correct they can go over their solution, try to find the mistakes, and submit another answer. However, I was not ready to give up critiquing the complete solutions as oppose to just checking the final answer - this is a very important aspect of students' learning.

It was clear that, if I use WeBWorK in my courses, I would have additional assignments (besides tests) on which I will grade and provide feedback. I first used WeBWorK in Calculus I four years ago. I continued to assign 10-20 practice problems from the textbook every class period; however, I did not collect nor grade these problems. Regardless, the students still solved these problems because every week I gave a short 15-minute quiz, with 2-3 problems, and the problems on these quizzes came directly from the list of the assigned practice problems. This way, I still provided feedback to the whole solution, checked my students' understand of the concepts, and gave them suggestions how to improve their mathematical writing.

I was pleased with the results that my students showed. Students appreciated WeBWorK because it eliminates paper-based homework; instead, they used their computers, tablets, and smart phones to complete the homework. They particularly liked that the system provided unlimited attempts for solving problems and encouraged them to work towards completion of all problems. When they got an incorrect answer, they went back to 
their solution looking for mistakes, they discussed the solutions with their peers, or came to my office hours to ask for help. I can, with great deal of confidence, say that WeBWorK increased the communication between the students, and between the students and myself; it also increased their confidence. At the same time, I was always able to monitor their progress and provide help if necessary. Using the WeBWorK problems the students mastered the techniques covered in the class and the weekly quizzes helped them improve the understanding of the concepts and improve their mathematical writing.

Since I first used WeBWorK in Calculus I four years ago, I have been using it every single time I teach any of the three Calculus courses in the same way as described above. I have not taught multiple sections of the same Calculus course in the same semester, so I could not conduct a study with one section in which I will use WeBWorK and a section (a control group) in which I will not use WeBWorK and compare the performance of the students. However, I have taught each of the three Calculus courses without the use of WeBWorK (before 2012) and I noticed that the students enrolled in the courses that used the online homework system performed significantly better on the tests. The grades were higher, in average, for about $30 \%$, the number of withdraws from the course and the number of students receiving a grade $\mathrm{F}$ was significantly reduced, for about $70 \%$. I know that comparing a performance of students now and more than four years ago does not provide a proper assessment of the effectiveness of the online homeworks. However, I, as a professor in these courses, noticed that the students learning Calculus using WeBWorK are better prepared for tests and exams, perform better on them, and are more motivated to learn mathematics than the students in the pre-WeBWorK years. Also, the students that used WeBWorK are more confident and better in communicating mathematics with their peers and myself.

At the end of each course, the students are asked to fill out course evaluations with various questions about the course, the delivery methods, the assessment methods, the instructor, etc. There is also a place for free responses on these evaluations. Whenever I have used WeBWorK for homework assignments, students addressed their opinions about it in these evaluations. Below are summery of their responses in which they mentioned WeBWorK.

(1) Almost all students believed that WeBWorK help them learn Calculus and they had a positive learning experience with it.

(2) Certainly all students liked the immediate feedback, and the unlimited attempts to solve the problems helped them learn the material better. They believed that having the opportunity to try to solve a 
problem multiple times encouraged them not to give up and actually learn how to do the problem. If it was a standard, paper and pencil homework assignment, they would try it once, and if the solution was not correct, they would be penalized and not learn from their incorrect answer.

(3) About a half of the students stated that they learned the most by trying to find mistakes in their solutions and correct them, or by discussing their incorrect solutions with their peers or myself.

(4) Majority of the students would like to use WeBWorK in other mathematics classes.

\section{WEBWORK IN OTHER CLASSES}

The online homework systems might successfully be used in other mathematics courses. I have also used WeBWorK in Linear Algebra I and Applied Statistics (only once in each course). When I used WeBWorK in Linear Algebra I, I taught the course using a modification of the flipped-classroom method. The students were asked to learn computational techniques on their own before coming to class. For example, they were asked to learn how to determine if a square matrix is invertible or not and if yes, find its inverse; to find a basis for a vector space; to determine if a set of vectors in a vector space/subspace is linearly dependent or independent; to find eigenvalues, eigenvectors, eigensubspaces, etc. So the students had to learn something "easy" on their own before coming to class. To make sure that they indeed learned the assigned techniques, they had to complete a WeBWorK assignment before coming to class. Then they were prepared for class, knew the elementary techniques and concepts, and we used the class period to discuss harder concepts and prove theorems. The Applied Statistics course in which I used WeBWorK was an online course, so WeBWorK really blended well with the instructional methods used in the course.

\section{REFERENCES}

[1] S. Bonham, R.Beichner, D. Deardorff, Online Homework: Does it Make a Difference?, Phys. Teach. 39, 293-296 (2001).

[2] M. Gage, A. Pizer, V. Roth, WeBWorK:Generating, delivering, and checking math homework via the Internet, ICTM2 International Congress for Teaching of Mathematics at the Undergraduate Level, Hersonissos, Crete, Greece (2002)

[3] http://webwork.maa.org

Department of Mathematics and Computer Science, Western Carolina UNIVERSITY, USA.

E-mail address: ratanasov@email.wcu.edu 